\title{
Landau levels in biased graphene structures with monolayer-bilayer interfaces
}

\author{
M. Mirzakhani, ${ }^{1,2, *}$ M. Zarenia, ${ }^{1, \dagger}$ P. Vasilopoulos, ${ }^{3, \dagger}$ S. A. Ketabi, ${ }^{2, \S}$ and F. M. Peeters ${ }^{1, \|}$ \\ ${ }^{1}$ Department of Physics, University of Antwerp, Groenenborgerlaan 171, B-2020 Antwerp, Belgium \\ ${ }^{2}$ School of Physics, Damghan University, P.O. Box 36716-41167, Damghan, Iran \\ ${ }^{3}$ Department of Physics, Concordia University, 7141 Sherbrooke West, Montreal, Quebec, Canada H4B 1R6
}

(Received 9 May 2017; revised manuscript received 25 July 2017; published 21 September 2017)

\begin{abstract}
The electron energy spectrum in monolayer-bilayer-monolayer and in bilayer-monolayer-bilayer graphene structures is investigated and the effects of a perpendicular magnetic field and electric bias are studied. Different types of monolayer-bilayer interfaces are considered as zigzag (ZZ) or armchair (AC) junctions which modify considerably the bulk Landau levels (LLs) when the spectra are plotted as a function of the center coordinate of the cyclotron orbit. Far away from the two interfaces, one obtains the well-known LLs for extended monolayer or bilayer graphene. The LL structure changes significantly at the two interfaces or junctions where the valley degeneracy is lifted for both types of junctions, especially when the distance between them is approximately equal to the magnetic length. Varying the nonuniform bias and the width of this junction-to-junction region in either structure strongly influence the resulting spectra. Significant differences exist between $\mathrm{ZZ}$ and AC junctions in both structures. The densities of states (DOSs) for unbiased structures are symmetric in energy whereas those for biased structures are asymmetric. An external bias creates interface LLs in the gaps between the LLs of the unbiased system in which the DOS can be quite small. Such a pattern of LLs can be probed by scanning tunneling microscopy.
\end{abstract}

DOI: 10.1103/PhysRevB.96.125430

\section{INTRODUCTION}

A family of two-dimensional (2D) graphene systems, consisting of one or a few layers of monolayer graphene (MLG), has attracted considerable attention during the last years because of their unique electronic properties that may be important for nanoelectronics applications [1-5]. MLG as well as bilayer graphene (BLG) has been prepared by several techniques, including precipitation on a silicon carbide surface [6,7], mechanical exfoliation from graphite [1,2], and growth by chemical vapor deposition (CVD) on catalytic metal surfaces [8-10].

Topographic images reveal that most multilayer samples exfoliated from graphite often contain atomic steps and islands of one or few layers of graphene [11-13]. Junctions between regions of different numbers of graphene layers can result in interesting properties. For instance, an unconventional Landau quantization was recently observed at the interface of such hybrid systems $[14,15]$. The transmission probability through a single MLG-BLG junction was calculated in the absence of a magnetic field [16], while in its presence the energy spectrum and local density of states of a semi-infinite MLG-BLG junction were studied in Ref. [17]. Transport properties of BLG with locally decoupled graphene sheets have also been investigated theoretically [18]. Another study was devoted to a hybrid quantum dot (QD) structure made of MLG-BLG junctions [19].

Motivated by the studies of Refs. [16] and [19], we investigate composite structures in which a narrow BLG ribbon

\footnotetext{
*mohamad.mirzakhani@gmail.com

†mohammad.zarenia@uantwerpen.be

${ }^{\ddagger}$ p.vasilopoulos@concordia.ca

§saketabi@du.ac.ir

"francois.peeters@uantwerpen.be
}

is sandwiched between MLG regions (Fig. 1) or a narrow MLG ribbon is sandwiched between BLG regions (Fig. 8). We consider double MLG-BLG junctions and investigate the effect of a perpendicular magnetic field and electric bias that was not included in Ref. [17]. Results for two types of junctions in either ribbon, with zigzag (ZZ) or armchair (AC) edges, are contrasted with each other. Unidirectional motion of interface states is predicted for such junctions.

Solving the Dirac-Weyl equation, we apply the appropriate boundary conditions at the junctions and obtain analytical results for the energy levels and corresponding wave functions. Our results show that the energy spectrum depends on the width of the MLG or BLG ribbons, the type of junctions, the size of the electric bias, and the strength of the magnetic field. The $K$ and $K^{\prime}$ valleys are studied separately because of the lack of inversion and time-reversal symmetries brought by the interfaces and the magnetic field, respectively.

The paper is organized as follows. In Sec. II we consider a MLG-BLG-MLG structure with ZZ and AC junctions and present analytical and numerical results. In Sec. III we do the same for a BLG-MLG-BLG structure, and in Sec. IV we present the corresponding densities of states. Our conclusions follow in Sec. V.

\section{MLG-BLG-MLG STRUCTURE}

We consider a BLG nanoribbon sandwiched between MLG regions, as shown in Fig. 1. This structure can be regarded as an infinite MLG sheet on which a second MLG ribbon is sitting on top of the first, thus realizing a BLG ribbon in the AB (Bernal) stacking configuration. For simplicity, we consider the ribbon to be infinite along the $y$ direction so that $k_{y}$ remains a good quantum number due to the translational symmetry along the $y$ direction.

To the left and right of the BLG ribbon and in the presence of a perpendicular magnetic field $\mathbf{B}=B \hat{e}_{z}$, the dynamics of 
(a) Zigzag

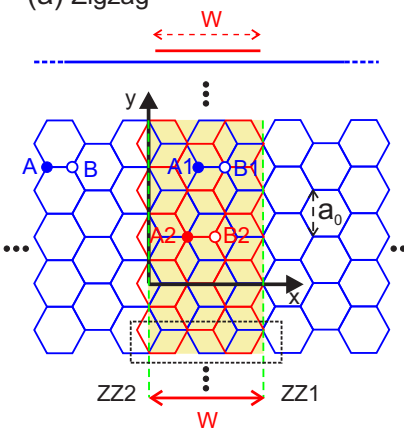

(b) Armchair

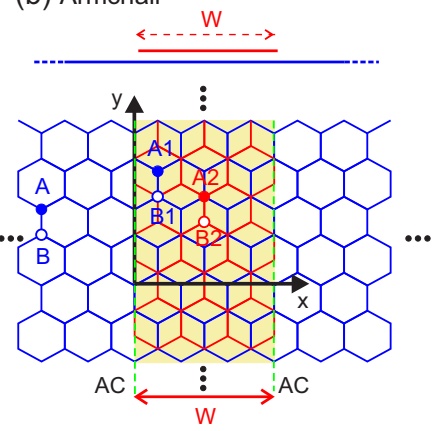

FIG. 1. Schematics of a MLG-BLG-MLG structure with (a) ZZ and (b) AC junctions. The BLG ribbon's width $W$ (yellow region) is $W=N \sqrt{3} a_{0} / 4$ in the $\mathrm{ZZ}$ case, with $a_{0}=0.246 \mathrm{~nm}$ the lattice constant and $N$ the number of atoms of the terminated layer in the black dashed box. In the AC case we have $W=n a_{0}$, with $n$ the number of lattice constants between the boundaries, i.e., the vertical green lines. The upper pictures show a side view of the systems.

the carriers in MLG, in the $K$ valley, is described by the Hamiltonian [20-22]

$$
\mathcal{H}^{K}=\left(\begin{array}{cc}
U_{1} & v_{F} \pi_{-} \\
v_{F} \pi_{+} & U_{1}
\end{array}\right),
$$

where $v_{F} \approx 10^{6} \mathrm{~m} / \mathrm{s}$ is the Fermi velocity, $\pi_{ \pm}=\pi_{x} \pm i \pi_{y}$ with $\boldsymbol{\pi}=-i \hbar \nabla+e \mathbf{A}$ the momentum operator, $\mathbf{A}=(0, B x, 0)$ is the vector potential in the Landau gauge, and $U_{1}$ the potential applied to MLG. The Hamiltonian at the $K^{\prime}$ valley is obtained by interchanging $\pi_{+}$with $\pi_{-}$in Eq. (1).

At the $K$ valley, the two-component envelope function $\Psi^{K}(\mathbf{r})$ satisfies the Schrödinger equation

$$
\mathcal{H}^{K} \Psi^{K}(\mathbf{r})=E \Psi^{K}(\mathbf{r}),
$$

with

$$
\Psi^{K}(\mathbf{r})=\left(\begin{array}{l}
\psi_{A}^{K}(x) \\
\psi_{B}^{K}(x)
\end{array}\right) e^{i k_{y} y},
$$

and $\psi_{A}^{K}$ and $\psi_{B}^{K}$ are the envelope functions on the sublattices $A$ and $B$, respectively. After some algebra we obtain

$$
v_{F} \pi_{+}=i E_{0} b^{\dagger}, \quad v_{F} \pi_{-}=-i E_{0} b,
$$

where $E_{0}=\sqrt{2} \hbar v_{F} / l_{B}$ and $l_{B}=\sqrt{\hbar / e B}$ is the magnetic length. Here, we have introduced the raising and lowering operators

$$
b^{\dagger}=-\partial / \partial z+z / 2, \quad b=\partial / \partial z+z / 2,
$$

where the dimensionless coordinate $z$ is defined by

$$
z=\sqrt{2}\left(x-x_{c}\right) / l_{B},
$$

and $x_{c}=-k_{y} l_{B}^{2}$ is the center of the cyclotron orbit.

The Schrödinger equation for the spinor components of the envelope function (3) becomes the coupled set of equations

$$
-i b \psi_{B}^{K}(z)=\varepsilon_{1} \psi_{A}^{K}(z), \quad i b^{\dagger} \psi_{A}^{K}(z)=\varepsilon_{1} \psi_{B}^{K}(z),
$$

where $\varepsilon_{1}=\varepsilon-u_{1}, \varepsilon=E / E_{0}$, and $u_{1}=U_{1} / E_{0}$. Decoupling the above equations leads to the Weber differential equation [23]

$$
\left(v-b^{\dagger} b\right) \psi_{B}^{K}=\left(\frac{\partial^{2}}{\partial z^{2}}+v+\frac{1}{2}-\frac{z^{2}}{4}\right) \psi_{B}^{K}(z)=0,
$$

with

$$
v=\left(\varepsilon-u_{1}\right)^{2} \text {. }
$$

The two independent solutions of Eq. (8) are the parabolic cylinder functions $D_{v}(z)$ and $D_{-v-1}(i z)$. The solution $D_{v}(z)$ converges to zero in the limit $z \rightarrow \infty$, while $D_{-v-1}(i z)$ diverges in both limits $z \rightarrow \pm \infty$. On the other hand, $D_{v}(-z)$ with the relation

$$
D_{\nu}(-z)=e^{i \nu \pi} D_{\nu}(z)+\frac{\sqrt{2 \pi}}{\Gamma(-v)} e^{i(v+1) \pi / 2} D_{-\nu-1}(i z)
$$

can also be chosen as an independent solution when $v \neq$ $0,1,2 \ldots$, which vanishes in the limit $z \rightarrow-\infty$. Therefore, we take $D_{v}(-z)$ in the region $x<0$ and $D_{v}(z)$ in the region $x>W$ as the solution for $\psi_{B}^{K}(z)$. The component $\psi_{A}^{K}(z)$ can be obtained from Eq. (7) with the help of the relations

$$
\begin{aligned}
b^{\dagger} D_{v}(z) & =\operatorname{sgn}(z) D_{v+1}(z), \\
b D_{v}(z) & =\operatorname{sgn}(z) v D_{v-1}(z),
\end{aligned}
$$

where $\operatorname{sgn}(z)$ is the sign function. The wave functions for the left $(L)$ and right $(R)$ MLG regions become

$$
\begin{aligned}
& \left(\begin{array}{l}
\psi_{A}^{K}(x) \\
\psi_{B}^{K}(x)
\end{array}\right)_{L}=C_{L}\left(\begin{array}{c}
i \varepsilon_{1} D_{v-1}(-z) \\
D_{v}(-z)
\end{array}\right), \\
& \left(\begin{array}{l}
\psi_{A}^{K}(x) \\
\psi_{B}^{K}(x)
\end{array}\right)_{R}=C_{R}\left(\begin{array}{c}
-i \varepsilon_{1} D_{v-1}(z) \\
D_{v}(z)
\end{array}\right),
\end{aligned}
$$

respectively, with $C_{L}$ and $C_{R}$ the normalization constants.

To obtain the Landau level (LL) energies of bulk MLG, the wave functions must vanish at $x \rightarrow \pm \infty$, which is satisfied by choosing $v$ to be a positive integer, i.e., $v(\varepsilon)=0,1,2, \ldots$. Then from Eq. (9) we get [17,24]

$$
E_{ \pm}= \pm E_{0} \sqrt{n}+U_{1}
$$

with $n=0,1,2, \ldots$ for the LLs of bulk MLG. The + and signs indicate the conduction and valence bands, respectively. It should be mentioned that the LL energies of bulk MLG are independent of the valleys $K$ and $K^{\prime}$, that is, we have a twofold valley degeneracy. Then the LL spectrum is fourfold degenerate if we take into account the twofold spin degeneracy.

The BLG region is described in terms of four sublattices, $A_{1}, B_{1}$ for the lower layer and $A_{2}, B_{2}$ for the upper layer. We only consider the coupling between two atoms stacked on top of each other, e.g., $B_{1}$ and $A_{2}$, and ignore the small contributions of the other interlayer couplings. In the vicinity of the $K$ valley, the effective Hamiltonian is [25,26]

$$
\mathcal{H}^{K}=\left(\begin{array}{cccc}
U_{1} & v \pi_{-} & 0 & 0 \\
v \pi_{+} & U_{1} & \gamma_{1} & 0 \\
0 & \gamma_{1} & U_{2} & v \pi_{-} \\
0 & 0 & v \pi_{+} & U_{2}
\end{array}\right),
$$

where $\gamma_{1} \approx 400 \mathrm{meV}$ is the nearest-neighbor interlayer hopping term, and $U_{1}$ and $U_{2}$ are the potentials at the two layers. For the $K^{\prime}$ point the Hamiltonian can be obtained by interchanging $\pi_{+}$and $\pi_{-}$in Eq. (15). 
Solving Eq. (2) for the four-component envelope function $\Psi^{K}(\mathbf{r})=\left(\psi_{A 1}^{K}, \psi_{B 1}^{K}, \psi_{A 2}^{K}, \psi_{B 2}^{K}\right)^{T} e^{i k_{y} y}$ and using the relations

$$
\begin{aligned}
b^{\dagger} D_{-v}(i z) & =i v D_{-v-1}(i z), \\
b D_{-v}(i z) & =-i D_{-v+1}(i z),
\end{aligned}
$$

we obtain

$$
\begin{aligned}
\left(\begin{array}{c}
\psi_{A 1}^{K} \\
\psi_{B 1}^{K} \\
\psi_{A 2}^{K} \\
\psi_{B 2}^{K}
\end{array}\right)=\sum_{\mu= \pm} C_{1 \mu}\left(\begin{array}{c}
-\left(\eta / \varepsilon_{1}\right) D_{-v_{\mu}}(i z) \\
\eta D_{-v_{\mu}-1}(i z) \\
\varepsilon_{2} D_{-v_{\mu}-1}(i z) \\
-\left(v_{\mu}+1\right) D_{-v_{\mu}-2}(i z)
\end{array}\right) \\
+C_{2 \mu}\left(\begin{array}{c}
-i \zeta D_{v_{\mu}-1}(z) \\
\eta D_{v_{\mu}}(z) \\
\varepsilon_{2} D_{v_{\mu}}(z) \\
i D_{v_{\mu}+1}(z)
\end{array}\right)
\end{aligned}
$$

where $\eta=\tilde{\gamma}_{1} \varepsilon_{1} \varepsilon_{2} /\left(\varepsilon_{1}^{2}-v_{\mu}\right), \zeta=\left(\nu_{\mu} \eta / \varepsilon_{1}\right), \tilde{\gamma}_{1}=\gamma_{1} / E_{0}$, and $\varepsilon_{1,2}=\varepsilon-u_{1,2}$, in which $u_{1,2}=U_{1,2} / E_{0}$, and

$$
v_{\mu}=\frac{1}{2}\left(\varepsilon_{+}^{2}-1+\mu\left[\varepsilon_{-}^{2}\left(\varepsilon_{-}^{2}+2\right)+4{\tilde{\gamma_{1}}}^{2} \varepsilon_{1} \varepsilon_{2}+1\right]^{1 / 2}\right) \text {. }
$$

$C_{1 \mu}$ and $C_{2 \mu}$ are normalization constants and $\varepsilon_{ \pm}^{2}=\varepsilon_{1}^{2} \pm \varepsilon_{2}^{2}$.

The condition of vanishing wave function at $x \rightarrow \pm \infty$ gives the LL energies of bulk BLG. This is only satisfied for $D_{v}(z)$ with integer $v \geqslant 0$. Therefore, the allowed values for the indices are $\nu_{+}(\varepsilon)=0,1,2, \ldots$ and $\nu_{-}(\varepsilon)=-1,0,1, \ldots$ independent of the valleys. Although for $v_{-}=-1,0$ and $v_{+}=0$ one can include $D_{v}(z)$ with negative indices, the corresponding coefficients are zero. In an unbiased system, i.e., for $U_{1}=U_{2}=0$, there are two zero-energy states with indices $v_{+}=0$ and $v_{-}=-1[17,26]$. Thus, the LL spectrum of BLG is fourfold degenerate (valley and spin degeneracy) except for the zero-energy LLs which are eightfold degenerate.

$$
M_{\mathrm{ZZ}}^{K}=\left(\begin{array}{ccc}
-\varepsilon_{1} D_{v-1}\left(-z_{1}\right) & 0 & i\left(\eta / \varepsilon_{1}\right) D_{-v_{+}}\left(i z_{1}\right) \\
-D_{v}\left(-z_{1}\right) & 0 & \eta D_{-v_{+}-1}\left(i z_{1}\right) \\
0 & 0 & \varepsilon_{2} D_{-v_{+}-1}\left(i z_{1}\right) \\
0 & \varepsilon_{1} D_{v-1}\left(z_{2}\right) & i\left(\eta / \varepsilon_{1}\right) D_{-v_{+}}\left(i z_{2}\right) \\
0 & -D_{v}\left(z_{2}\right) & \eta D_{-v_{+}-1}\left(i z_{2}\right) \\
0 & 0 & i\left(v_{+}+1\right) D_{-v_{+}-2}\left(i z_{2}\right)
\end{array}\right.
$$

\section{A. Zigzag junctions}

As illustrated in Fig. 1(a), for the ZZ junctions of a MLG-BLG-MLG structure, it is not possible to have the same edge interface at both sides, and therefore, it always includes a pair of different ZZ boundaries, ZZ1 and ZZ2. At the ZZ1 boundary $[x=W$ in Fig. 1(a)], the outermost atoms are from the $A 2$ sublattice, which is directly coupled to $B 1$ via $\gamma_{1}$, while at the ZZ2 boundary $[x=0$ in Fig. 1(a)] the outermost atoms are $B 2$. The wave functions corresponding to the sublattices in the lower layer are continuous at the boundaries, while the $\mathrm{ZZ}$ boundary condition implies that the wave spinors corresponding to the terminated atoms in the upper layer become zero [16]. Accordingly, at $x=0$, the boundary conditions for ZZ2 are

$$
\begin{aligned}
\psi_{A 1}^{v}\left(z_{1}\right) & =\psi_{A}^{v}\left(z_{1}\right)_{L}, \\
\psi_{B 1}^{v}\left(z_{1}\right) & =\psi_{B}^{v}\left(z_{1}\right)_{L}, \\
\psi_{A 2}^{v}\left(z_{1}, y\right) & =0,
\end{aligned}
$$

where $v=K$ or $K^{\prime}$ and $z_{1}=-\sqrt{2} x_{c} / l_{B}$. At $x=W$ we have

$$
\begin{aligned}
& \psi_{A 1}^{v}\left(z_{2}\right)=\psi_{A}^{v}\left(z_{2}\right)_{R}, \\
& \psi_{B 1}^{v}\left(z_{2}\right)=\psi_{B}^{v}\left(z_{2}\right)_{R}, \\
& \psi_{B 2}^{v}\left(z_{2}\right)=0,
\end{aligned}
$$

for the ZZ1 boundary with $z_{2}=\sqrt{2}\left(W-x_{c}\right) / l_{B}$. The conditions (19) and (20) lead to a system of equations from which we obtain the eigenvalues by setting the determinant of the coefficients equal to zero. Then using Eqs. (12), (13), and (17) leads for the $K$ valley to

$$
M_{\mathrm{ZZ}}^{K}\left(C_{L}, C_{R}, C_{1+}, C_{1-}, C_{2+}, C_{2-}\right)^{T}=0,
$$

where $T$ denotes the transpose of the row vector and

$$
\begin{aligned}
& -\zeta D_{v_{+}-1}\left(z_{1}\right) \\
& \quad \eta D_{v_{+}}\left(z_{1}\right) \\
& \varepsilon_{2} D_{v_{+}}\left(z_{1}\right) \\
& -\zeta D_{v_{+}-1}\left(z_{2}\right) \\
& \quad \eta D_{v_{+}}\left(z_{2}\right) \\
& D_{v_{+}+1}\left(z_{2}\right)
\end{aligned}
$$$$
\left.\begin{array}{c}
-\zeta D_{v_{-}-1}\left(z_{1}\right) \\
\eta D_{v_{-}}\left(z_{1}\right) \\
\varepsilon_{2} D_{v_{-}}\left(z_{1}\right) \\
-\zeta D_{v_{-}-1}\left(z_{2}\right) \\
\eta D_{v_{-}}\left(z_{2}\right) \\
D_{v_{-}+1}\left(z_{2}\right)
\end{array}\right)
$$

A similar matrix, $M_{\mathrm{ZZ}}^{K^{\prime}}$, is obtained for the $K^{\prime}$ valley. The eigenvalues are obtained from the conditions $\operatorname{det}\left|M_{\mathrm{ZZ}}^{K}\right|=0$ and det $\left|M_{\mathrm{ZZ}}^{K^{\prime}}\right|=0$.

The zero-energy states can be obtained separately by solving the Schrödinger equation $\mathcal{H}^{K} \Psi^{K}(\mathbf{r})=0$ for $U_{1}=$ $U_{2}=0$ in the MLG and BLG regions. The details of the derivation for the zero energies of a MLG-BLG junction were discussed in Refs. [16] and [17]. These zero-energy levels are due to edge states and to the zeroth LLs in bulk MLG or BLG LLs. The results are summarized in Table I, which shows the degeneracy of the zero-energy levels in the MLG and BLG regions for each boundary. We used the notation $+n$ to indicate the additional degeneracy of these levels due to edge states. For the $K\left(K^{\prime}\right)$ valley, due to the ZZ2 edge, the additional zero energy appears on the left MLG side in the limit $x \rightarrow-\infty$, (BLG and the right MLG side when $x \rightarrow+\infty)$, and on the other hand, the edge of ZZ1 causes another zero-energy state in BLG and on the left MLG side in the limit $x \rightarrow-\infty$ (in the right MLG side for $x \rightarrow+\infty$ ). Therefore, as shown in Table I, the MLG regions contain a total of $2+2$ zero-energy states in the limits of $x \rightarrow \pm \infty$, two of which are bulk MLG LLs and +2 edge states due to the ZZ1 and ZZ2 edges. For a wide BLG ribbon there are $4+2$ zero-energy states, containing four bulk BLG LLs and +2 edge states due to the ZZ1 and ZZ2 edges (the spin degrees are neglected).

It is interesting to note the difference between the present interface states in MLG-BLG heterostructures and the snake states arising at the $p-n$ junction in the presence of a perpendicular magnetic field [27]. In the latter the particle 
TABLE I. Number of zero-energy LLs in each part of the MLG-BLG-MLG system for ZZ (upper panel) and AC (lower panel) junctions in the limits $x_{c}= \pm \infty$, and in the assumption of a wide-width BLG region in which the energy states inside converge to the BLG LLs. The zero-energy edge states are indicated by $+n$.

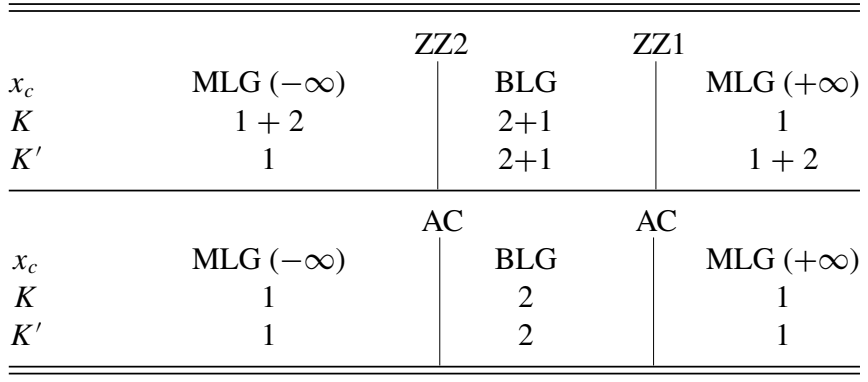

transits from an electron into a hole and vice versa when crossing the $p-n$ junction.

Figures 2(a)-2(c) show the energy levels of an unbiased structure $\left(U_{1}=U_{2}=0\right)$ for $B=10 \mathrm{~T}$ as a function of $x_{c}$ for three different BLG widths, (a) $W_{1}=71.58 \mathrm{~nm}$, (b) $W_{2}=47.72 \mathrm{~nm}$, and (c) $W_{3}=11.93 \mathrm{~nm}$, in the case of $\mathrm{ZZ}$ boundaries for electrons in the $K$ (blue solid curves) and $K^{\prime}$ (red dashed curves) valleys. The energy levels merge into the Landau levels of bulk MLG in the limits $x_{c} \rightarrow \pm \infty$ for both valleys. In a wide BLG ribbon, such as that of Fig. 2(a), we have $W_{1}(71.58 \mathrm{~nm}) \gg l_{B}(=8.10 \mathrm{~nm})$ and the energy levels inside the BLG ribbon approach the LLs of bulk BLG for low energies. The horizontal gray lines inside the yellow region [obtained from Eq. (18)] show the LLs of bulk BLG. Near the junctions, breaking of the inversion symmetry due to the interface removes the degeneracy of the $K$ and $K^{\prime}$ valleys. Because of the different ZZ1 and ZZ2 boundary conditions at the left and right junctions, the spectrum is asymmetric with respect to the center of the BLG ribbon. The plateaulike and oscillatory features that appear in the spectrum near the interfaces can be understood as the hybridization of energy levels of the terminated system, semi-infinite MLG and BLG.

Decreasing the width of the BLG ribbon [see Figs. 2(b) and $2(\mathrm{c})$ ] prevents the energy levels inside the ribbon from
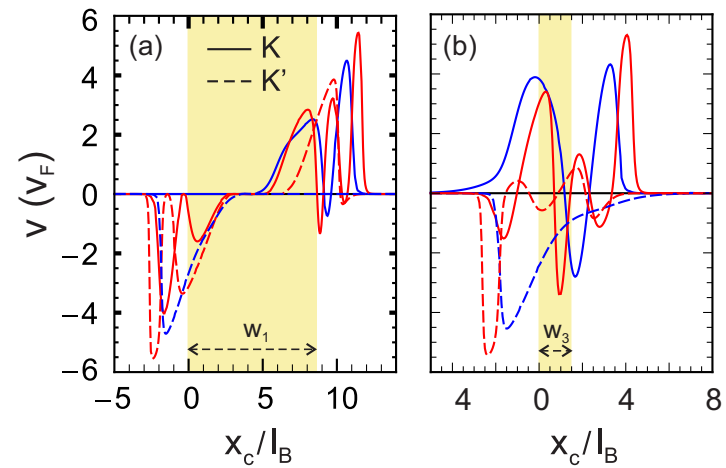

FIG. 3. Electron velocity along the interfaces as a function of $x_{c} / l_{B}$, for the two lowest electron states of the spectrum shown in (a) Fig. 2(a) and (b) Fig. 2(c) at the $K$ (solid curves) and $K^{\prime}$ (dashed curves) valleys. The blue and red curves are for the first and second energy states, respectively.

converging to the BLG LLs due to the overlap of the wave functions of the edges. When the width of the ribbon is comparable with the magnetic length $l_{B}$, e.g., $W_{3}=11.93 \mathrm{~nm} \approx l_{B}=8.10$ $\mathrm{nm}$, the value of the energy levels inside the ribbon increase in comparison with those of wide ribbons, and the levels show a more oscillatorylike behavior through the ribbon [see Fig. 2(c)]. It should be mentioned that the electron-hole symmetry is preserved for both valleys because all matrix elements in $M_{\mathrm{ZZ}}^{K}$ [Eq. (22)] and $M_{\mathrm{ZZ}}^{K^{\prime}}$ are independent of the sign of the energy $\varepsilon$ when $U_{1}=U_{2}=0$. For the $K\left(K^{\prime}\right)$ valley, as Fig. 2(a) shows, the $n$th $[(n+1)$ th] MLG LLs connect to the $n$th BLG LLs at $x=0$ with a ZZ2 boundary, and at $x=W_{1}$ with a ZZ1 boundary, the $n$th BLG LLs approach the $(n+1)$ th ( $n$ th) LLs of MLG. This indicates that due to the presence of the ribbon, the $n$th $[(n+1)$ th] LLs of the left MLG finally connect to the $(n+1)$ th $(n$ th) LLs of the right MLG for the $K$ $\left(K^{\prime}\right)$ valley.

Figures 3(a) and 3(b) show the $y$ component of the carrier velocity, in units of $v_{F}$, for the first two states of the spectrum in Figs. 2(a) and 2(c), respectively. The solid (dashed) curves are for the $K\left(K^{\prime}\right)$ valley. Electrons localized near the interface
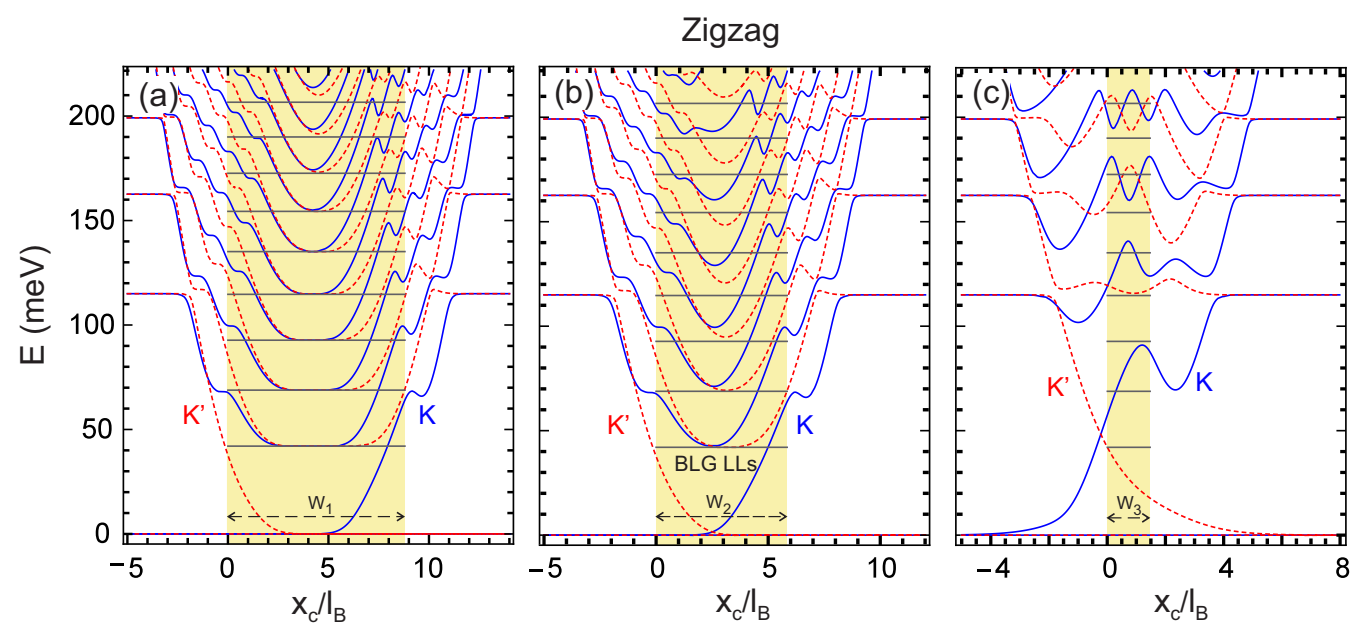

FIG. 2. Energy spectrum of an unbiased MLG-BLG-MLG structure $\left(U_{1}=U_{2}=0\right)$ versus $x_{c} / l_{B}=-k_{y} l_{B}$, with ZZ junctions in a magnetic field $B=10 \mathrm{~T}$, for three different widths of the BLG ribbon (yellow region): (a) $W_{1}=71.58 \mathrm{~nm}$, (b) $W_{2}=47.72 \mathrm{~nm}$, and (c) $W_{3}=11.93 \mathrm{~nm}$. Blue solid (red dashed) curves refer to the $K\left(K^{\prime}\right)$ valley. 

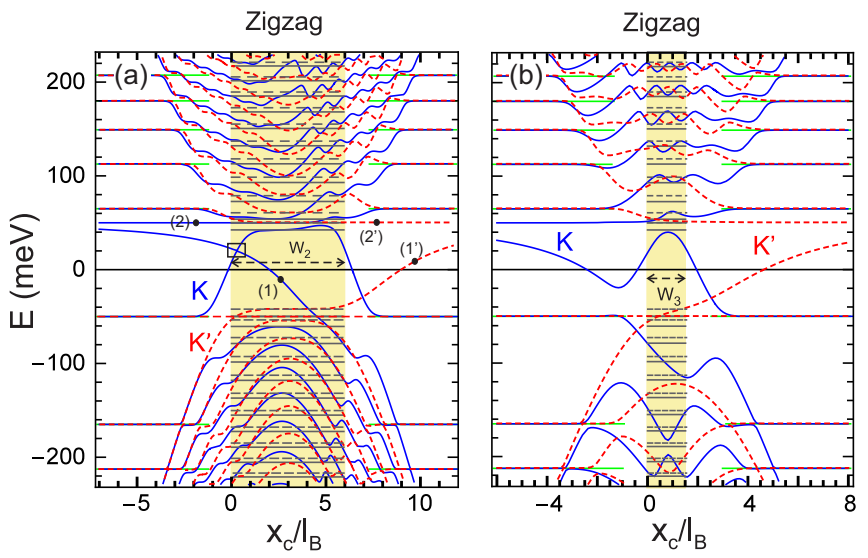

FIG. 4. Energy spectrum of a biased MLG-BLG-MLG structure $\left(U_{1}=-U_{2}=-50 \mathrm{meV}\right)$ as a function of $x_{c} / l_{B}$, with $\mathrm{ZZ}$ boundaries in the presence of a perpendicular magnetic field $B=10 \mathrm{~T}$, for BLG ribbon width (yellow region) (a) $W_{2}=47.72 \mathrm{~nm}$ and (b) $W_{3}=11.93 \mathrm{~nm}$. Blue solid (red dashed) curves display the energy spectrum at the $K\left(K^{\prime}\right)$ valley. Degenerate LLs of MLG are shown by green solid lines, and gray solid (dashed) lines indicate the LLs of biased BLG for the $K\left(K^{\prime}\right)$ valley.

propagate along the edges of the BLG ribbon and move in opposite directions at the right and left interfaces. For the first $K-\left(K^{\prime}-\right)$ energy state that merges into the zero-energy level in MLG, the electrons move only near the right (left) edge. As the width of the BLG ribbon becomes narrower, due to the overlap of the edge states, the electrons have nonzero velocity when located inside the ribbon.

Results for the spectrum of a biased structure, $U_{1}=-U_{2}=$ $-50 \mathrm{meV}$, are plotted in Fig. 4 for a BLG ribbon of width (a) $W_{2}=47.72 \mathrm{~nm}$ and (b) $W_{3}=11.93 \mathrm{~nm}$. As can be seen, the electron-hole symmetry is broken due to the broken inversion symmetry induced by the external gate potential. As expected, the energy levels approach the biased MLG LLs [green solid lines obtained from Eq. (14)] in the limits $x_{c} \rightarrow \pm \infty$ for both valleys. For a wide BLG ribbon, e.g., $W_{2}$, the energy levels approach the biased BLG LLs at low energies which are nondegenerate [28] for the valleys $K$ (gray solid lines) and $K^{\prime}$ (gray dashed lines). The LLs of biased BLG for the $K$ valley can be obtained from Eq. (18). At high energies, as well as in the case of narrow ribbons, e.g., $W_{3}$, the energy levels show an oscillatory behavior and coincide no longer with the biased BLG LLs. Furthermore, the point surrounded by the black rectangular box in Fig. 4(a) is an anticrossing point that disappears upon decreasing the BLG ribbon width, as is obvious from Fig. 4(b). The levels indicating the energy points labeled by $(1),(2)$ and $\left(1^{\prime}\right),\left(2^{\prime}\right)$ in Fig. 4(a) are the edge states at the $K$ and $K^{\prime}$ valleys, respectively. These states have a chiral character localized at the MLG-BLG interfaces.

Figure 5 shows the probability densities for each sublattice, $\left|\psi_{i}\right|^{2}(i=A, B, \ldots)$, corresponding to the points labeled (1), $(2),\left(1^{\prime}\right)$, and $\left(2^{\prime}\right)$ in the energy spectrum of Fig. 4 . The solid and dashed curves show the probability density for layers 1 and 2, respectively, and their continuity at the boundaries obviously results from that of the wave functions. The points (1) and ( $\left.1^{\prime}\right)$, which belong to the $K$ and $K^{\prime}$ valleys, respectively, indicate a strong confinement at the ZZ1 boundary while points (2) and $\left(2^{\prime}\right)$ indicate a confinement mostly at the ZZ2 boundary. On the other hand, the edge states at the boundaries ZZ1 and ZZ2 are mostly concentrated at the $A 2$ and $B 2$ sites, respectively.

\section{B. Armchair junctions}

We consider AC boundaries as shown in Fig. 1(b). Although two types of $\mathrm{AC}$ boundaries exist, the boundary conditions are the same at both interfaces [16], namely,

$$
\begin{aligned}
\psi_{A 1}^{v}(z) & =\psi_{A}^{v}(z), \\
\psi_{B 1}^{v}(z) & =\psi_{B}^{v}(z), \\
\psi_{A 2}^{K}(z)-\psi_{A 2}^{K^{\prime}}(z) & =0, \\
\psi_{B 2}^{K}(z)+\psi_{B 2}^{K^{\prime}}(z) & =0,
\end{aligned}
$$

where $z$ is given by Eq. (6). The last two equations indicate that intervalley mixing occurs at the AC boundary. Applying the conditions (23) at $x=0$ and $x=W$ leads to the determinant $\operatorname{det}\left|M_{A C}\right|=0$ of a $12 \times 12 M_{A C}$ matrix. As indicated in Table I, there is no edge state in the AC junction, and each region contains only its own bulk zero LLs, i.e., two zero-energy states for the MLG regions and four for the wide BLG ribbon.

The energy levels as a function of $x_{c}$ are shown in Fig. 6 for an unbiased structure and three different widths of the BLG ribbon: (a) $W_{1}=71.34 \mathrm{~nm}$, (b) $W_{2}=48.22 \mathrm{~nm}$, and (c) $W_{3}=12.30 \mathrm{~nm}$ in a field $B=10 \mathrm{~T}$. Applying the same boundary condition at $x=0$ and $x=W$ renders the spectrum symmetric. In the limits $x_{c} \rightarrow \pm \infty$ the energy levels approach the doubly degenerate bulk MLG LLs. With their degeneracy lifted near the junctions, the levels merge into the BLG LLs inside the wide BLG ribbon [see Fig. 6(a)]. Decreasing the width of the ribbon removes the degeneracy of the levels inside
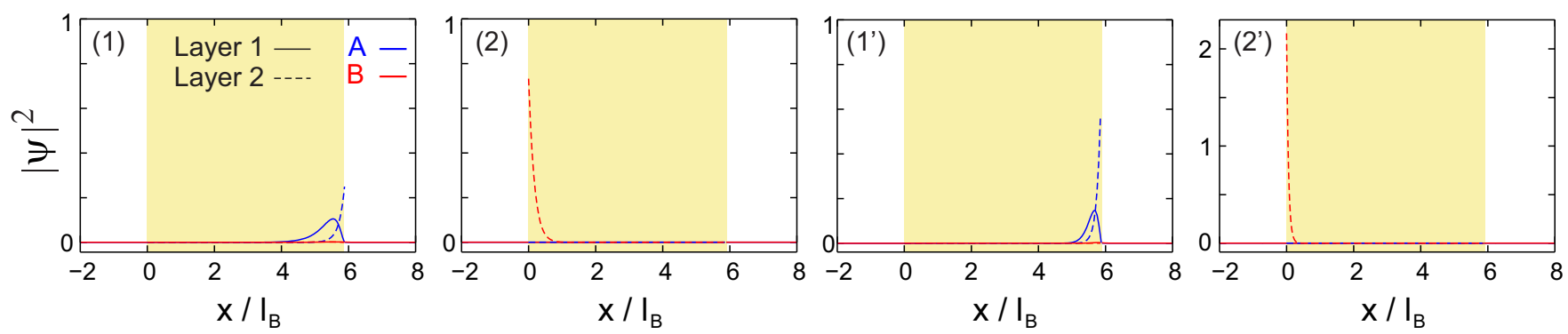

FIG. 5. Probability densities for each sublattice corresponding to the points (1), (2), (1'), and (2') shown in the energy spectrum of Fig. 4(a). The solid and dashed curves are for sites in layers 1 and 2, respectively, and the blue (red) color for sublattice $A(B)$. 

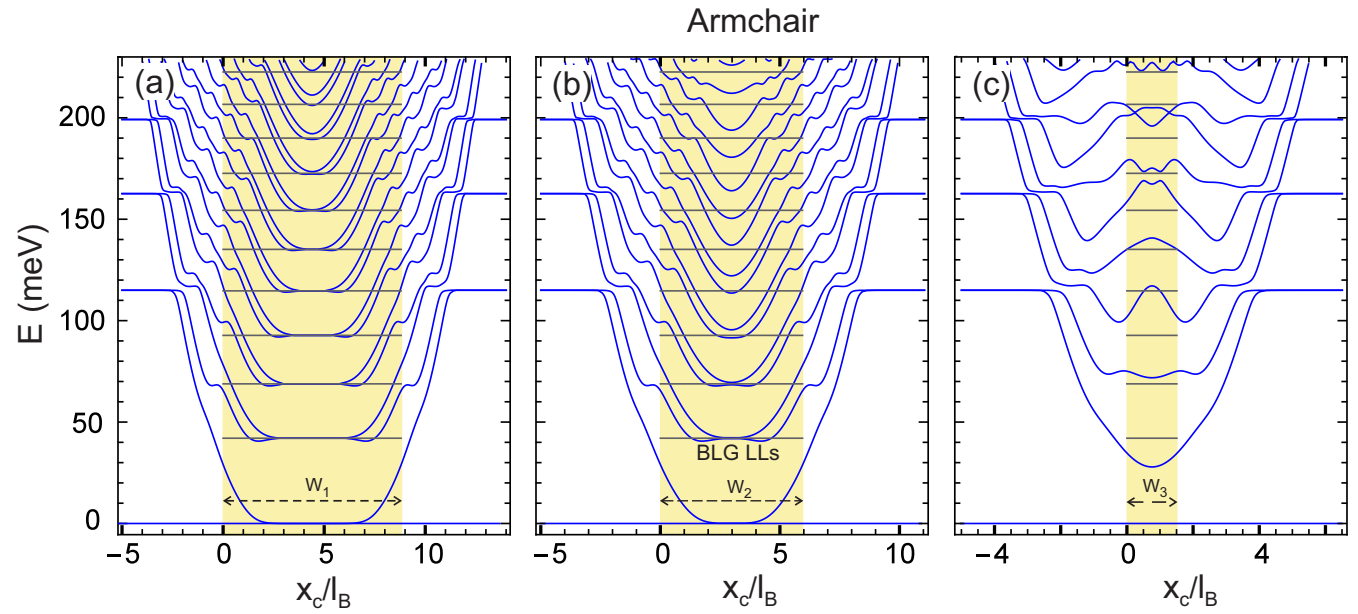

FIG. 6. Energy spectrum of an unbiased MLG-BLG-MLG structure $\left(U_{1}=U_{2}=0\right)$ versus $x_{c} / l_{B}$ with AC junctions in a magnetic field $B=10 \mathrm{~T}$ for three different widths of the BLG ribbon (yellow region): (a) $W_{1}=71.34 \mathrm{~nm}$, (b) $W_{2}=48.22 \mathrm{~nm}$, and (c) $W_{3}=12.30 \mathrm{~nm}$. The gray solid lines are the BLG LLs.

it and opens a gap between the zero-energy and the first excited state [Fig. 6(c)]. The electron-hole symmetry is also preserved for the AC boundary.

Figure 7 shows the energy levels of a biased MLG-BLGMLG structure $\left(U_{1}=-U_{2}=-50 \mathrm{meV}\right)$ with AC junctions. The width of the BLG ribbon is (a) $W_{2}=48.22 \mathrm{~nm}$ and (b)
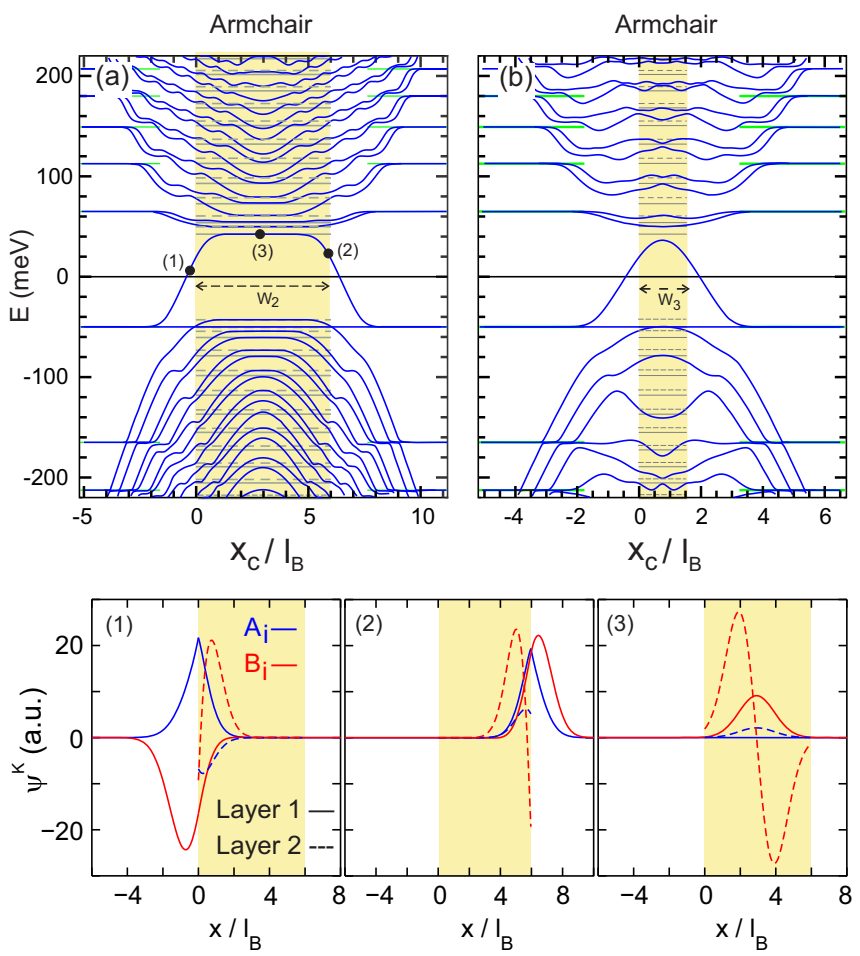

FIG. 7. (Upper panels) Energy levels of a biased MLG-BLGMLG structure $\left(U_{1}=-U_{2}=-50 \mathrm{meV}\right)$, as a function of $x_{c} / l_{B}$, with AC boundaries in a magnetic field $B=10 \mathrm{~T}$. The width of the BLG ribbon is (a) $W_{2}=48.22 \mathrm{~nm}$ and (b) $W_{3}=12.30 \mathrm{~nm}$. The green lines show the degenerate MLG LLs, and the gray solid (dashed) ones are the $K\left(K^{\prime}\right)$-valley LLs of BLG. The lower panels show the spinors of each sublattice corresponding to points (1)-(3) in panel (a).
$W_{3}=12.30 \mathrm{~nm}$, and the applied magnetic field is $B=10 \mathrm{~T}$. The external gate potential breaks the inversion symmetry and results in the electron-hole asymmetry seen in Fig. 7. The spectrum in Fig. 7(a) shows two intermediate chiral states, i.e., localized at the left and right interfaces (see lower panels), connecting the first hole LL of MLG to the first electron LL in BLG. For a small width of BLG ribbon [Fig. 7(b)], these interface states overlap and prevent carriers to be confined inside the BLG ribbon. Similar to the ZZ boundary, the energy levels converge to the biased MLG LLs, Eq. (14), in the limits $x_{c} \rightarrow \pm \infty$. At low energies, as is evident from Fig. 7(a), the energy levels merge into the biased BLG LLs, which are nondegenerate for the $K$ (gray solid lines) and $K^{\prime}$ (gray dashed lines) valleys inside the BLG ribbon. The wave functions corresponding to the $K$-valley energy states (1)-(3) in the spectrum of Fig. 7(a) are shown in the lower panels of Fig. 7. As expected, states (1) and (2) show carrier localization on the left and right interfaces, respectively, and the lowest spinor amplitude belongs to the $A 2$ sublattice, which is a dimer site subjected to the potential $U_{2}=50 \mathrm{meV}$. On the other hand, the state (3), belonging to the lowest biased BLG LL, is confined inside the BLG (yellow) ribbon and is mostly concentrated on the $B 2$ sublattice. Notice that the spinors corresponding to the LLs are symmetric.

\section{BLG-MLG-BLG STRUCTURE}

Below, we consider a narrow MLG ribbon sandwiched by AB-stacked BLG regions, which, as shown in Fig. 8, is the inverse of the situation investigated in Sec. II. This structure can be regarded as an infinite BLG sheet in which a ribbon of width $W$ is cut out from its upper layer, thus creating the MLG ribbon. The physical assumptions are the same as in Sec. II. Similar to that case, the Hamiltonian is solved for the MLG and BLG parts, and then the appropriate wave functions are chosen for the left and right semi-infinite BLG regions, which decay exponentially for $x \rightarrow \pm \infty$. 
(a) Zigzag

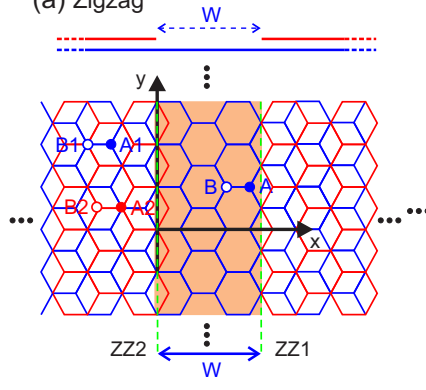

(b) Armchai

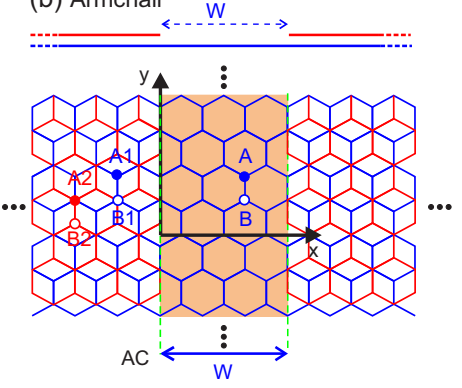

FIG. 8. Schematics of a BLG-MLG-BLG structure with (a) ZZ and (b) AC junctions. $W$ is the width of the MLG region. The upper pictures show a side view of the systems.

\section{A. Zigzag junctions}

Figure 8(a) shows schematically the $\mathrm{ZZ}$ junctions for a BLG-MLG-BLG structure. After solving the Schrödinger equation [Eq. (2)] for the MLG and BLG regions and applying the ZZ boundary conditions (19) and (20) at $x=0$ and $x=W$, respectively, we obtain the corresponding matrices for the two valleys $K$ and $K^{\prime}$. To avoid repetition, we only present the numerical results. The results for the degeneracy of the zero-energy states are summarized in Table II. Here, too, $+n$ indicates the additional degeneracy resulting from edge states.

The energy spectrum of an unbiased structure $\left(U_{1}=U_{2}=\right.$ $0)$ versus $x_{c}$ is shown in Fig. 9 for three different widths of the MLG ribbon, (a) $W_{1}=71.58 \mathrm{~nm}$, (b) $W_{2}=47.72 \mathrm{~nm}$, and (c) $W_{3}=11.93 \mathrm{~nm}$, at the two valleys $K$ (blue solid curves) and $K^{\prime}$ (red dashed curves) with $B=10 \mathrm{~T}$. As expected, the energy levels approach the LLs of bulk BLG for $x_{c} \rightarrow \pm \infty$ and converge to the MLG LLs inside the widest ribbon shown in Fig. 9(a). For the widest ribbon, the spectrum shows plateaulike (in ZZ2 boundary) and oscillatory (in ZZ1 boundary) features similar to the semi-infinite MLG-BLG junction [17]. Decreasing the width of MLG ribbon causes the wave functions of edges to couple with each other and the spectrum to show only an oscillatory feature through the ribbon (see below). Further, as seen in Fig. 9(c), the spectrum for the $K^{\prime}$ valley shows an oscillatory behavior with strong anticrossings at low energies and near the MLG ribbon, while the $K$-valley spectrum shows just oscillatory behavior. It is

TABLE II. Number of zero-energy LLs for each part of a BLGMLG-BLG structure at the ZZ (top) and AC (bottom) junctions for $x \rightarrow \pm \infty$, with the condition that the MLG ribbon is sufficiently wide for its energy levels to converge to the MLG LLs. The zero-energy edge states are indicated by $+n$.

\begin{tabular}{|c|c|c|c|c|}
\hline & & $\mathrm{ZZ2}$ & $\mathrm{ZZ1}$ & \\
\hline$x_{c}$ & $\operatorname{BLG}(-\infty)$ & MLG & & $\operatorname{BLG}(+\infty)$ \\
\hline$K$ & 2 & $1+1$ & & $2+2$ \\
\hline \multirow[t]{2}{*}{$K^{\prime}$} & $2+2$ & $1+1$ & & 2 \\
\hline & & $\mathrm{AC}$ & $\mathrm{AC}$ & \\
\hline$x_{c}$ & $\operatorname{BLG}(-\infty)$ & MLG & & $\operatorname{BLG}(+\infty)$ \\
\hline$K$ & 2 & 1 & & 2 \\
\hline$K^{\prime}$ & 2 & 1 & & 2 \\
\hline
\end{tabular}

also worth noting that there are more (oscillatory) energy levels inside the MLG ribbon in this BLG-MLG-BLG structure than in the BLG ribbon of the MLG-BLG-MLG structure, cf. Figs. 2(c) and 9(c).

In the lower panels of Fig. 9, we plot the wave-function components for the energies labeled (1)-(6) in Figs. 9(b) and 9(c). For a wide ribbon [Fig. 9(b)], the states near the boundaries are strongly confined close to the interfaces and in the BLG regions [cf. Figs. 9(1) and 9(2)]. Inside the ribbon and right at the MLG LLs, the carriers are mostly confined in the ribbon, as point (3) shows. As the width of the ribbon decreases, the edge states corresponding to the left [of state (4)] and the right [of state (5)] interfaces overlap and exhibit confinement on both sides of the BLG ribbon. Right at the anticrossing, see point (6), the overlap is strong and hence the spinors have a large amplitude at both boundaries. Points (1)-(6) show that the spinors of A1 and B2 atoms possess large amplitudes, since the low-energy spectrum of BLG is dominated by those nondimer sites, cf. Fig. 8 .

Figure 10 shows the energy spectrum of a biased structure with a MLG ribbon width (a) $W_{2}=47.72 \mathrm{~nm}$ and (b) $W_{3}=$ $11.93 \mathrm{~nm}$ for the two valleys $K$ (blue solid curves) and $K^{\prime}$ (red dashed curves). In the BLG regions, the energy levels, in the limits $x \rightarrow \pm \infty$, show a gap between the valence and conduction bands and coincide with the nondegenerate biased BLG LLs [gray solid $(K)$ and dashed $\left(K^{\prime}\right)$ lines]. Near the interfaces and beyond the gap, the energy levels of each valley with different BLG LL indices show oscillatory feature and finally merge into the (degenerate) biased MLG LLs (green solid lines) with the same indices at low energies and for a wide MLG ribbon, see Fig. 10(a). Decreasing the width of the MLG ribbon results in the appearance of more energy levels inside the ribbon, as seen in Fig. 10(b). Further, in both valleys, the electron states show a smooth oscillatory and strong anticrossings are visible for the hole states.

\section{B. Armchair junction}

The AC junction is shown schematically in Fig. 8(b). Similar to Sec. II B, we obtain the corresponding $12 \times 12$ matrix for this $\mathrm{AC}$ junction. Below, we present only the numerical results.

The energy spectra, as a function of $x_{c}$, are shown in Fig. 11 for three different widths of the MLG ribbon, (a) $W_{1}=71.58 \mathrm{~nm}$, (b) $W_{2}=47.72 \mathrm{~nm}$, and (c) $W_{3}=11.93$ $\mathrm{nm}$, in a field $B=10 \mathrm{~T}$ with $U_{1}=U_{2}=0$. As mentioned in Table II, there is no edge state at the AC boundary. The spectra are symmetric due to the same boundary conditions applied at $x=0$ and $x=W$. In the limits $x_{c} \rightarrow \pm \infty$, the energy levels are twofold degenerate and agree with the LLs of BLG. Near the interfaces, the LL degeneracy is lifted and each of the levels converge to a different LL in the MLG ribbon due to the breaking of the inversion symmetry. For the wide MLG ribbon [Fig. 11(a)] the energy levels converge to the MLG LLs. For a narrow MLG ribbon, of width comparable to the magnetic length, e.g., $W_{3}=12.30 \mathrm{~nm} \approx l_{B}=8.10 \mathrm{~nm}$, the energy levels increase in number and show an oscillatory behaviour with anticrossings inside the ribbon [see Fig. 11(c)].

Figure 12 shows the energy levels of a biased structure $\left(U_{1}=-U_{2}=-50 \mathrm{meV}\right)$ with a MLG ribbon width of (a) 

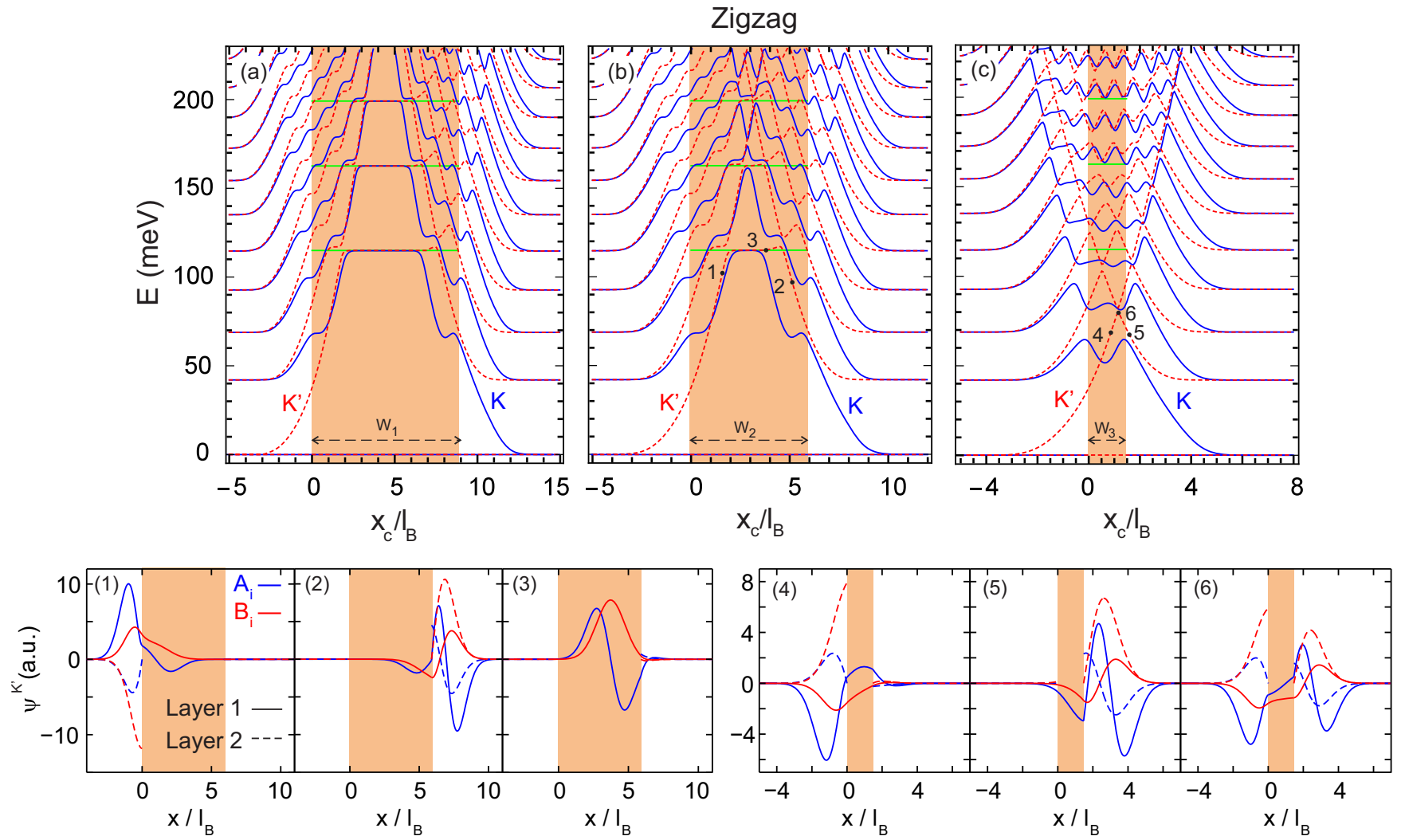

FIG. 9. (Upper panels) Energy spectrum of an unbiased BLG-MLG-BLG structure $\left(U_{1}=U_{2}=0\right)$ as a function of $x_{c} / l_{B}$, with ZZ boundaries in a field $B=10 \mathrm{~T}$, for three different MLG ribbon widths (brown regions): (a) $W_{1}=71.58 \mathrm{~nm}$, (b) $W_{2}=47.72 \mathrm{~nm}$, and (c) $W_{3}=11.93 \mathrm{~nm}$. The blue solid (red dashed) curves refer to the $K\left(K^{\prime}\right)$ valley. The LLs in MLG are shown by the green solid lines. The lower panels show the states for the energies marked by (1)-(6) in panels (b) and (c).

$W_{2}=48.22 \mathrm{~nm}$ and (b) $W_{3}=12.30 \mathrm{~nm}$, in a field $B=10 \mathrm{~T}$. The general properties of the unbiased case [see Figs. 11(b) and 11(c)] remain unchanged except that now (i) the breaking of the inversion symmetry breaks the electron-hole symmetry, (ii) an energy gap opens between the electron and hole energy levels in the MLG ribbon, and (iii) the energy levels coincide with the nondegenerate biased BLG LLs [gray solid $(K)$ and dashed $\left(K^{\prime}\right)$ lines] at $x_{c} \rightarrow \pm \infty$.

\section{DENSITY OF STATES}

We now consider the density of states for the studied structures which can be probed by quantum capacitance measurements or also using a scanning tunneling microscopy (STM). It is given by

$$
D(E)=\frac{D_{0}}{2 \pi} \sum_{n} \int d k_{y}^{\prime} \delta\left(\varepsilon-\varepsilon_{n, k_{y}}\right),
$$

where $D_{0}=1 / \sqrt{2} \hbar v_{F}$ and $k_{y}^{\prime}=x_{c} / l_{B}$. To numerically evaluate $D(E)$, we replace the $\delta$ function by a Gaussian of width $\Gamma_{1}=0.025 \mathrm{meV}$ and $\Gamma_{2}=0.005 \mathrm{meV}$, respectively, for a MLG-BLG-MLG and a BLG-MLG-BLG structure. Both widths are smaller than the corresponding energy-level separation.

Figure 13 shows the DOS as a function of the energy. Panels (a), (b) are for a MLG-BLG-MLG structure and panels (c), (d) for a BLG-MLG-BLG one. The upper and lower panels are for
$\mathrm{ZZ}$ (with the ribbon width of $W_{2}=47.72 \mathrm{~nm}$ ) and $\mathrm{AC}$ (with $W_{2}=48.22 \mathrm{~nm}$ ) junctions, respectively.

The blue curves are for an unbiased structure and the red ones for a biased one with $U_{1}=-U_{2}=-50 \mathrm{meV}$. Due to the electron-hole symmetry, the DOS for unbiased structures is symmetric in energy whereas that for biased structures is
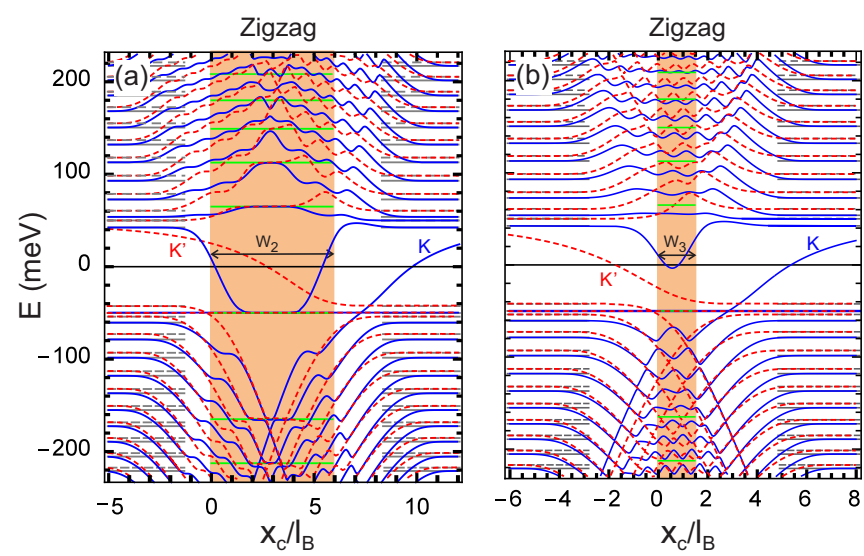

FIG. 10. Energy spectrum of a biased BLG-MLG-BLG structure $\left(U_{1}=-U_{2}=-50 \mathrm{meV}\right.$ ) versus $x_{c} / l_{B}$, with $\mathrm{ZZ}$ boundaries in a field $B=10 \mathrm{~T}$. The MLG width (brown region) is (a) $W_{2}=47.72 \mathrm{~nm}$ and (b) $W_{3}=11.93 \mathrm{~nm}$. The bulk MLG and BLG LLs are shown in green and gray solid lines, respectively. 

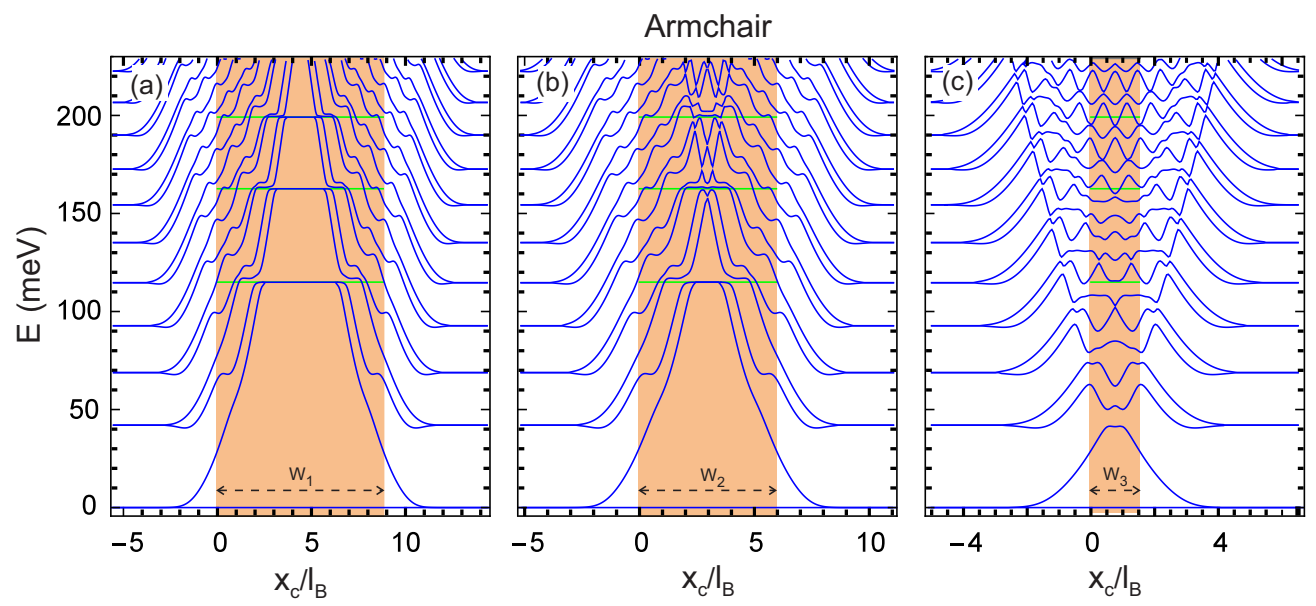

FIG. 11. Energy spectrum of an unbiased BLG-MLG-BLG structure $\left(U_{1}=U_{2}=0\right)$, as a function of $x_{c} / l_{B}$, with AC boundaries in a magnetic field $B=10 \mathrm{~T}$. The width of the MLG ribbon (brown region) is (a) $W_{1}=71.34 \mathrm{~nm}$, (b) $W_{2}=48.22 \mathrm{~nm}$, and (c) $W_{3}=12.30 \mathrm{~nm}$. The solid green lines are the LLs of bulk MLG.

asymmetric. The sharp peaks correspond to the LLs of the extended MLG and BLG sheets. The MLG LL peaks in panels (a) and (b) are more pronounced than the BLG LL ones shown in panels (c) and (d). Due to the finite range taken for $k_{y}^{\prime}$ and the broadening of the $\delta$ function, the normally infinitely high peaks at the LLs appear finite. In the unbiased BLG-MLGBLG structure we observe peaks with energy $E \approx \pm 115 \mathrm{meV}$, which include the LLs both of the BLG sheet and the MLG ribbon, see also Figs. 9(b) and 11(b). Small peaks, seen in all panels, correspond, in each system, to low-energy LLs of either the ribbon or interface LLs (plateaulike regions) appearing in the spectra.

When a bias is present the sharp peaks are shifted in a MLGBLG-MLG structure; contrast panels (a) and (b). In a BLGMLG-BLG structure, though, only the peaks corresponding to the lowest LLs are shifted and the others are almost unaffected; contrast panels (c) and (d).
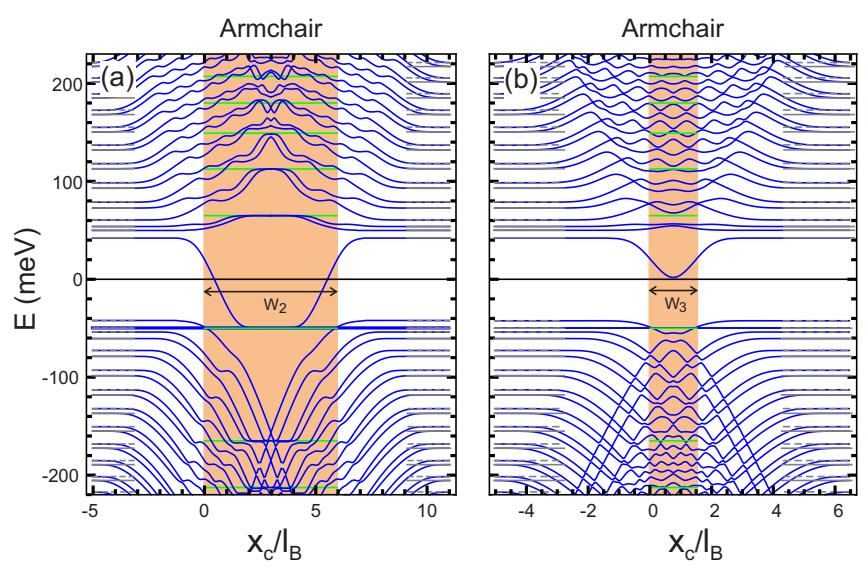

FIG. 12. Energy spectrum of a biased BLG-MLG-BLG structure $\left(U_{1}=-U_{2}=-50 \mathrm{meV}\right)$, versus $x_{c} / l_{B}$, with $\mathrm{AC}$ boundaries in a magnetic field $B=10 \mathrm{~T}$. The width of the MLG ribbon is (a) $W_{2}=$ $48.22 \mathrm{~nm}$ and (b) $W_{3}=12.30 \mathrm{~nm}$.
To probe the interface states by STM, the states must lie in a low-DOS region. In unbiased MLG-BLG-MLG and BLG-MLG-BLG structures with a magnetic field $B=10 \mathrm{~T}$, considered in this work, this condition is satisfied. The DOS is high at the LLs and low in the gaps between LLs. For instance, in clean samples, LL broadening due to disorder and interaction effects is less than $\sim 2 \mathrm{meV}[29,30]$. For biased MLG-BLG-MLG structure, it is experimentally possible to probe the pattern of the states between the lowest MLG LLs. The appearance of nondegenerate $K$ and $K^{\prime}$ BLG LLs (which are close to each other at low potentials) in combination with any LL broadening in real biased BLG-MLG-BLG structures makes the probing of the interface states difficult. Increasing the magnetic field results in large LL gaps and enables one to overcome this difficulty.
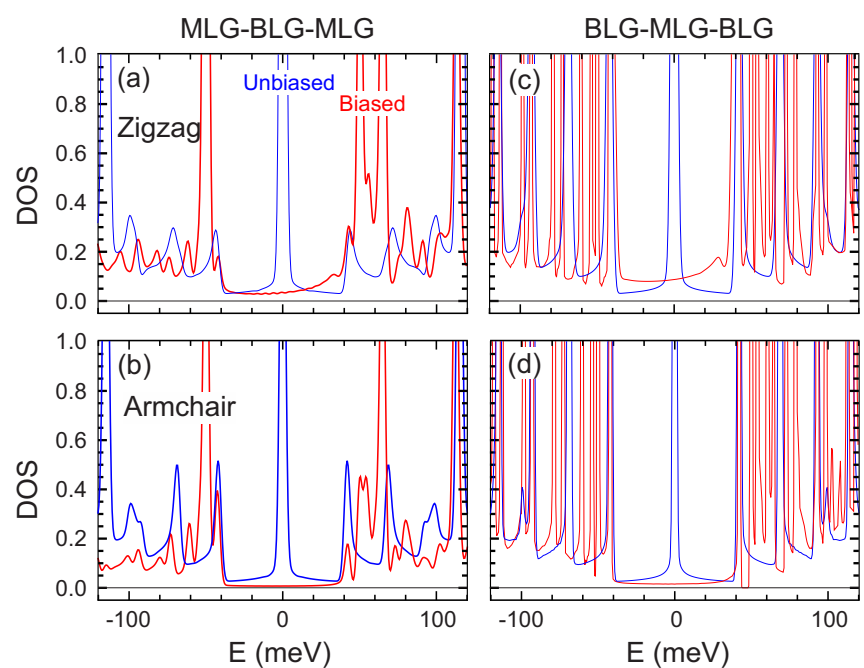

FIG. 13. Density of states in MLG-BLG-MLG and BLG-MLGBLG structures for ZZ (upper panels with $W_{2}=47.72 \mathrm{~nm}$ ) and AC (lower panels with $W_{2}=48.22 \mathrm{~nm}$ ) junctions. The blue curves pertain to an unbiased structure and the red ones to a biased one with $U_{1}=$ $-U_{2}=-50 \mathrm{meV}$. 


\section{CONCLUSIONS}

In summary, we extended the previous study on the interface Landau levels in a semi-infinite monolayer-bilayer graphene system [17] and investigated the influence of a finite-size BLG (MLG) ribbon surrounded by MLG (BLG) regions in the absence and presence of a perpendicular electric field. We considered both zigzag and armchair boundaries at the MLG-BLG junctions. We showed that two line interfaces at MLG-BLG junctions create two additional zero-energy edge states at the interfaces for zigzag boundaries (compared to one zero-energy edge state with one line interface in Ref. [17]). An oscillatory behavior of the energy spectrum also appears near the interfaces with zigzag boundaries and is most pronounced for widths $W_{3}$ of the order of the magnetic length that cannot be obtained with one line interface. Our results also show that interface states are localized at either side of the ribbon and overlap as the ribbon becomes narrower, which prevents forming BLG (MLG) LLs inside the BLG (MLG) ribbon. These interface states exhibit a unidirectional motion along the interface which can have a group velocity larger than $v_{F}$.

In the presence of a bias potential that induces a perpendicular electric field in the BLG region, intermediate chiral states are localized at the MLG-BLG interfaces. Further, in the biased BLG, the valley degeneracy of the LLs is lifted. By controlling the width of the BLG (or MLG) ribbon or the type of boundaries, these intermediate states and their subsequent magnetic response can be configured. In the case of zigzag interfaces, the chiral states corresponding to the $K$ and $K^{\prime}$ valleys are confined at opposite sides of the ribbon, suggesting potential applications. Such chiral states are related to similar states that have been observed in different BLG systems, e.g., twisted BLG and BLG subjected to an asymmetric gate potential.

Without any external potential, the DOS is symmetric with respect to electrons and holes and exhibits a large peak at zero energy brought by the zigzag interfaces. The electron-hole symmetry is broken in biased systems, and LL shifts occur for both structures. An external bias creates interface LLs in the LL gaps of the unbiased system in which the DOS can be quite small. Accordingly, it should be possible to experimentally probe this pattern of LLs by STM [31].

Although for realistic samples the structure would be more complex than that modeled here, we believe that the main features of our near analytical results can be captured in relevant experimental systems, e.g., the interface LLs in MLG-BLG planar junctions have been experimentally realized using scanning tunneling spectroscopy measurements $[14,15]$. We emphasize that to study a realistic complex system of interfaces in few-layer graphene samples one could adopt a tight-binding approach, but then the calculations would be limited to very small sizes, in contrast with our near analytical results obtained by the continuum approach.

\section{ACKNOWLEDGMENTS}

This work was supported by the BOF-UA (Bijzonder Onderzoeks Fonds), the Canadian NSERC through Grant No. OGP0121756 (P.V.), and the Methusalem Program of the Flemish Government.
[1] K. S. Novoselov, A. K. Geim, S. V. Morozov, D. Jiang, Y. Zhang, S. V. Dubonos, I. V. Grigorieva, and A. A. Firsov, Science 306, 666 (2004).

[2] K. S. Novoselov, E. McCann, S. V. Morozov, V. I. Fal'ko, M. I. Katsnelson, U. Zeitler, D. Jiang, F. Schedin, and A. K. Geim, Nat. Phys. 2, 177 (2006).

[3] Y.Zhang, Y.-W. Tan, H. L. Stormer, and P. Kim, Nature (London) 438, 201 (2005).

[4] K. Chung, C.-H. Lee, and G.-C. Yi, Science 330, 655 (2010).

[5] G. Jo, M. Choe, C.-Y. Cho, J. H. Kim, W. Park, S. Lee, W.-K. Hong, T.-W. Kim, S.-J. Park, B. H. Hong, Y. H. Kahng, and T. Lee, Nanotechnology 21, 175201 (2010).

[6] C. Berger, Z. Song, X. Li, X. Wu, N. Brown, C. Naud, D. Mayou, T. Li, J. Hass, A. N. Marchenkov, E. H. Conrad, P. N. First, and W. A. de Heer, Science 312, 1191 (2006).

[7] T. Ohta, A. Bostwick, T. Seyller, K. Horn, and E. Rotenberg, Science 313, 951 (2006).

[8] K. S. Kim, Y. Zhao, H. Jang, S. Y. Lee, J. M. Kim, K. S. Kim, J.-H. Ahn, P. Kim, J.-Y. Choi, and B. H. Hong, Nature (London) 457, 706 (2009)

[9] A. Reina, X. Jia, J. Ho, D. Nezich, H. Son, V. Bulovic, M. S. Dresselhaus, and J. Kong, Nano Lett. 9, 30 (2008).

[10] L. G. De Arco, Y. Zhang, A. Kumar, and C. Zhou, IEEE Trans. Nanotechnol. 8, 135 (2009).
[11] Y. Kobayashi, K. I. Fukui, T. Enoki, K. Kusakabe, and Y. Kaburagi, Phys. Rev. B 71, 193406 (2005).

[12] Y. Niimi, T. Matsui, H. Kambara, K. Tagami, M. Tsukada, and H. Fukuyama, Phys. Rev. B 73, 085421 (2006).

[13] G. M. Rutter, J. N. Crain, N. P. Guisinger, P. N. First, and J. A. Stroscio, J. Vac. Sci. Technol., A 26, 938 (2008).

[14] W. Yan, S.-Y. Li, L.-J. Yin, J.-B. Qiao, J.-C. Nie, and L. He, Phys. Rev. B 93, 195408 (2016).

[15] L.-J. Yin, K.-K. Bai, W.-X. Wang, S.-Y. Li, Y. Zhang, and L. He, Front. Phys. 12, 127208 (2017).

[16] T. Nakanishi, M. Koshino, and T. Ando, Phys. Rev. B 82, 125428 (2010).

[17] M. Koshino, T. Nakanishi, and T. Ando, Phys. Rev. B 82, 205436 (2010).

[18] H. M. Abdullah, M. Zarenia, H. Bahlouli, F. M. Peeters, and B. Van Duppen, Europhys. Lett. 113, 17006 (2016).

[19] M. Mirzakhani, M. Zarenia, S. A. Ketabi, D. R. da Costa, and F. M. Peeters, Phys. Rev. B 93, 165410 (2016).

[20] C. W. J. Beenakker, Rev. Mod. Phys. 80, 1337 (2008).

[21] P. Recher, J. Nilsson, G. Burkard, and B. Trauzettel, Phys. Rev. B 79, 085407 (2009).

[22] P. Recher and B. Trauzettel, Nanotechnology 21, 302001 (2010).

[23] Tables of Integrals, Series, and Products, 6th ed., edited by I. S. Gradshteyn and I. M. Ryzhik (Academic Press, San Diego, CA, 2000), p. 989. 
[24] E. McCann, in Graphene Nanoelectronics: Metrology, Synthesis, Properties and Applications (Springer Verlag, Berlin Heidelberg, 2011), p. 237.

[25] I. Snyman and C. W. J. Beenakker, Phys. Rev. B 75, 045322 (2007).

[26] E. McCann and V. I. Fal'ko, Phys. Rev. Lett. 96, 086805 (2006).

[27] M. Zarenia, J. M. Pereira, F. M. Peeters, and G. A. Farias, Phys. Rev. B 87, 035426 (2013).
[28] Y.-H. Ho, S.-J. Tsai, M.-F. Lin, and W.-P. Su, Phys. Rev. B 87, 075417 (2013).

[29] C. H. Yang, F. M. Peeters, and W. Xu, Phys. Rev. B 82, 075401 (2010).

[30] A. Pound, J. P. Carbotte, and E. J. Nicol, Phys. Rev. B 84, 085125 (2011).

[31] E. Y. Andrei, G. Li., and X. Du, Rep. Prog. Phys. 75, 056501 (2012). 